\title{
Oxidised Low-Density Lipoprotein Concentrations and Physical Activity Status in Older Adults: The WASEDA Active Life Study
}

\author{
Jong-Hwan Park ${ }^{1}$, Masashi Miyashita ${ }^{2}$, Masaki Takahashi ${ }^{1}$, Kazuhiro Harada ${ }^{1,3}$, Kanae Takaizumi ${ }^{1,3}$, \\ Hyun-Shik Kim ${ }^{1}$, Katsuhiko Suzuki ${ }^{2}$, and Yoshio Nakamura ${ }^{2}$
}

${ }^{1}$ Graduate School of Sport Sciences, Waseda University, Saitama, Japan
${ }^{2}$ Faculty of Sport Sciences, Waseda University, Saitama, Japan
${ }^{3}$ Japan Society for the Promotion of Science, Tokyo, Japan

Aim: Oxidised low-density lipoprotein (LDL) is considered a risk marker for cardiovascular disease. However, it remains unclear whether oxidised LDL concentrations differ with the physical activity status in older adults. The purpose of this study was to examine the relationship between the amount of physical activity and oxidised LDL in older adults.

Methods: Twenty-seven older adults (aged 69.9 \pm 4.3 years, mean \pm SD.; 16 female and 11 male) were analysed in the cross-sectional design. Prior to blood collection, participants were asked to wear an uniaxial accelerometer for 4 consecutive weeks to determine the physical activity status. After a $48-\mathrm{h}$ period of physical activity avoidance and a 10 -h overnight fast, fasting venous blood samples were obtained from each participant.

Results: Fasting plasma oxidised LDL concentrations and plasma monocyte chemoattractant protein- 1 concentrations were negatively correlated with the amount of physical activity $(r=-0.409$, $p=0.034 ; r=-0.385, p=0.047$, respectively).

Conclusions: This study demonstrates that regular physical activity may play a protective role in the oxidation of LDL in older adults.

J Atheroscler Thromb, 2011; 18:568-573.

Key words; Physical activity status, Oxidised low-density lipoprotein, Older adults, Cardiovascular disease

\section{Introduction}

Oxidised low-density lipoprotein (LDL) is taken up by macrophages via scavenger receptors, leading to foam cell formation and is considered a risk marker for cardiovascular disease ${ }^{1)}$. Furthermore, oxidised LDL has been shown to play an important role in the pathogenesis of atherosclerosis ${ }^{1,2)}$. Previous clinical studies have associated elevated oxidised LDL concentrations with the severity of acute coronary syndromes $^{3)}$ or coronary heart disease ${ }^{4)}$. In addition, observational studies have shown that oxidised LDL is

Address for correspondence: Masashi Miyashita, Faculty of Sport Sciences, Waseda University, 2-579-15 Mikajima, Tokorozawa, Saitama 359-1192, Japan

E-mail: m.miyashita@aoni.waseda.jp

Received: November 21, 2010

Accepted for publication: January 11, 2011 associated with an increased incidence of metabolic syndrome $^{5)}$ or increased number of components of metabolic syndrome ${ }^{6}$.

A previous cross-sectional study has reported that aerobically trained middle-aged individuals exhibit lower oxidised LDL concentrations than sedentary middle-aged individuals ${ }^{7}$. An intervention study has also demonstrated that aerobic exercise training decreased oxidised LDL concentrations in middleaged individuals with coronary artery disease ${ }^{8)}$. Furthermore, a previous study has shown that acute bouts of prolonged exercise reduced oxidised LDL concentrations in healthy young men ${ }^{9)}$. While these efficacy studies provide a protective role for regular physical activity against the oxidation of LDL and hence cardiovascular disease, it remains unclear whether oxidised LDL concentrations differ with the physical activity status in "older" adults. It is important to 
address this issue because circulating concentrations of oxidised LDL increase with age ${ }^{2,10)}$.

\section{Aim}

The purpose of the present study was to examine whether oxidised LDL concentrations differ with the physical activity status in older adults. We hypothesised that regularly active older individuals exhibit lower oxidised LDL concentrations.

\section{Methods}

\section{Participants}

After approval from the Waseda University Ethics Committee, 45 older adults aged $\geq 60$ (27 female and 18 male) gave their written informed consent to participate in the WASEDA Active Life study. Participants were recruited from the general populations (i.e. none were trained athletes competing in any sporting events but some participants were recreationally active) of two local communities (i.e. Tokorozawa and Iruma). To support local community health promotion, we accepted all participants to enroll this study if they corresponded to the study's criteria for having no difficulties in activities of daily living. In this crosssectional study, however, 18 participants were excluded from data analysis (but not from feedback). The reasons for exclusion included taking lipidand/or glucose-lowering medication, having inadequate wear time of an accelerometer (see "Physical Activity Measurement"), and performing strenuous physical activities in the 48 hours preceding the blood collection day. Thus, 27 participants' data (16 female and 11 male, aged of 61-80 years) are presented here. The physical characteristics of the participants are shown in Table 1.

\section{Physical Activity Measurement}

For the determination of physical activity levels, participants were asked to wear an uniaxial accelerometer (Lifecoder-EX; Suzuken Co. Ltd., Nagoya, Japan) for 4 consecutive weeks. Participants were contacted by phone at the beginning of each week to assess adherence and any device problems. No participants reported any problems with the use of the accelerometer. We also noted that all participants wore the accelerometer every day during the data collection period. We collected accelerometer data from all participants simultaneously, indicating that seasonality was not an issue in the present study. By measuring the magnitude and frequency of accelerations, the device determines the level of activity intensity (i.e. 11 levels: 0,
Table 1. Physical characteristics of the participants

\begin{tabular}{lc}
\hline Age (years) & $69.9 \pm 4.3(61-80)$ \\
Height $(\mathrm{m})$ & $1.56 \pm 0.07(1.38-1.70)$ \\
Body mass $(\mathrm{kg})$ & $53.2 \pm 8.7(34.7-72.3)$ \\
Body mass index $\left(\mathrm{kg} / \mathrm{m}^{2}\right)$ & $21.6 \pm 2.4(15.5-26.2)$ \\
Waist circumference $(\mathrm{cm})$ & $80.8 \pm 8.6(64.0-95.2)$ \\
Systolic blood pressure $(\mathrm{mmHg})$ & $128 \pm 8(115-148)$ \\
Diastolic blood pressure $(\mathrm{mmHg})$ & $72 \pm 8(60-98)$ \\
\hline
\end{tabular}

All data are presented as the means \pm SD (range); $n=27$

$0.5,1-9 ; 0$ is the lowest activity, 9 is the highest activity) every 4 seconds. Data from participants who had worn the accelerometer for at least 10 hours (600 minutes) a day for at least 4 weekdays and 1 weekend day (i.e. in total) after calculation of wear time were considered valid ${ }^{11,12)}$. The main physical activity variable used in this study was the time spent in moderate to vigorous physical activity (MVPA). Aerobic activities such as walking, dancing or cycling that cause increased heart rate and possibly sweating are usually above the threshold for moderate intensity activity. More strenuous activities, such as jogging and hill walking, would usually be classified as vigorous. MVPA was calculated on a daily basis, and then used to estimate weekly activity by taking a weighted average of daily weekday and weekend activity (i.e. weekly $\mathrm{MVPA}=($ average daily weekday MVPA $\times 5)+($ average daily weekend MVPA $\times 2)$ ). All minutes of recording with $\geq 4$ activity levels were classified as MVPA. The threshold of 4 activity level was derived from a calibration study ${ }^{13)}$ and corresponded to approximately 3 metabolic equivalents.

\section{Anthropometry}

Body mass was measured to the nearest $0.05 \mathrm{~kg}$ using a digital scale (Inner Scan 50; Tanita Corporation, Tokyo, Japan). Height was measured to the nearest $0.1 \mathrm{~cm}$ using a wall-mounted stadiometer (YS-OA; As One Corporation, Osaka, Japan). Body mass index was calculated as weight in kilograms divided by the square of height in meters. Waist circumference was measured to the nearest $0.1 \mathrm{~cm}$ at the level of the umbilicus using a flexible plastic tape while the participants were in the standing position.

\section{Arterial Blood Pressure}

Arterial blood pressure was measured from the right arm in a seated position by a standard mercury sphygmomanometer (605P; Yagami Co. Ltd., Nagoya, Japan). Participants were seated on a chair for $5 \mathrm{~min}$ utes before measurements. Two measurements were 
taken at each time point and the mean was recorded.

\section{Blood Collection and Laboratory Assays}

After a 48-h period of physical activity avoidance, fasting (i.e. after an overnight fast of at least 10 hours) venous blood samples were taken from an antecubital vein. Blood samples were analysed for measurement of plasma oxidised LDL, monocyte chemoattractant protein-1 (MCP-1), glucose, insulin, leptin, C-reative protein (CRP), soluble vascular cell adhesion molecule-1 (sVCAM-1), apolipoprotein(a) and adiponectin, and serum total cholesterol (TC), triacylglycerol, high-density lipoprotein cholesterol (HDL-C) and low-density lipoprotein cholesterol (LDL-C). For TC, triacylglycerol, HDL-C and LDL-C, the samples were collected into tubes containing clotting activators for isolation of serum. Thereafter, samples were allowed to clot for 45 minutes at room temperature and then centrifuged at $3000 \mathrm{rpm}$ for 10 minutes at $4{ }^{\circ} \mathrm{C}$. After separation, serum was dispensed into plain micro tubes and stored at $-80^{\circ} \mathrm{C}$ for later analysis. For plasma oxidised LDL, MCP-1, insulin, leptin, CRP, sVCAM-1, apolipoprotein(a) and adiponectin, venous blood samples were collected into tubes containing disodium salt-EDTA. Thereafter, samples were immediately centrifuged and treated as above. For plasma glucose measurements, venous blood samples were collected into tubes containing sodium fluoride-EDTA. Thereafter, samples were immediately centrifuged and treated as above.

Concentrations of plasma oxidised LDL (Mercodia AB, Uppsala, Sweden), MCP-1 (R\&D Systems Inc., Minneapolis, USA), insulin (Mercodia AB), leptin (R\&D Systems Inc.), CRP (R\&D Systems Inc.), sVCAM-1 (Mercodia AB), apolipoprotein(a) (eBioscience, Inc., San Diego, USA) and adiponectin (Sekisui Medical Co. Ltd., Tokyo, Japan) were measured by enzyme-linked immunosorbent assay using commercially available kits. Concentrations of serum TC, triacylglycerol, HDL-C, LDL-C, and plasma glucose were determined by standard laboratory methods.

\section{Statistical Analysis}

Data were analysed using the Predictive Analytics Software (PASW) version 18.0 for Windows (SPSS Japan Inc., Tokyo, Japan). The Shapiro-Wilk test was used to check for normality of distribution. The distribution of all parameters was found not to differ significantly from normal. The Pearson product moment correlation coefficient was used to examine relationships between variables. Stepwise multiple regression was used to assess the contribution of significantly associated variables identified by univariate regression analysis to oxidised LDL concentrations. For all variables included in the model, the variance inflation factors were $<1.9$, suggesting that multicollinearity was not a problem. Statistical significance was accepted at the 5\% level. Results are presented as the means \pm SD.

\section{Results}

The amount of MVPA determined by the accelerometer was $173.2 \pm 14.6 \mathrm{~min} /$ week (range: 62.6$312.9 \mathrm{~min} /$ week). Daily step counts of the participants were $9515 \pm 518$ steps/day (range: 3799-14075 steps/day).

Fasting concentrations of plasma oxidised LDL were inversely related to the amount of MVPA ( $r=$ $-0.409, p=0.034)$ (Fig. 1). A significant inverse association was observed between the amount of MVPA and fasting concentrations of plasma MCP-1 $(r=$ $-0.385, p=0.047$ ) (Fig. 2). A significant positive association was also observed between fasting concentrations of plasma oxidised LDL and fasting concentrations of plasma sVCAM-1 $(r=0.391, p=0.044)$. Fasting concentrations of plasma oxidised LDL concentrations were positively correlated with fasting concentrations of serum LDL concentrations $(r=0.391, p=$ $0.043)$.

Table 2 shows the relationships between the amount of MVPA and other blood parameters. The amount of MVPA was significantly correlated with fasting serum triacylglycerol $(r=-0.415, p=0.031)$, fasting serum HDL-C $(r=0.405, p=0.036)$ or fasting plasma insulin $(r=-0.482, p=0.011)$ concentrations. The amount of MVPA was also significantly correlated with body mass $(r=-0.444, p=0.020)$, body mass index $(r=-0.413, p=0.032)$ or waist circumference $(r=-0.528, p=0.005)$. No correlations were observed between the amount of MVPA and fasting serum TC, fasting serum LDL-C, fasting plasma glucose, fasting plasma leptin, fasting plasma CRP, fasting plasma sVCAM-1, fasting plasma apolipoprotein(a) or fasting plasma adiponectin concentrations. In multiple regression analyses with fasting plasma oxidised LDL concentrations as the dependent variable and the amount of MVPA, fasting serum HDL-C concentrations, fasting serum LDL-C concentrations, fasting serum triacylglycerol concentrations as the independent variables, fasting serum LDL-C and triacylglycerol concentrations together explained $43 \%$ of the variance in fasting plasma oxidised LDL concentrations (fasting serum LDL-C concentrations, standardised $\beta=0.572, p=0.002$; fasting serum triacylglycerol concentrations, standardised $\beta=0.545, p=0.028$ ). 


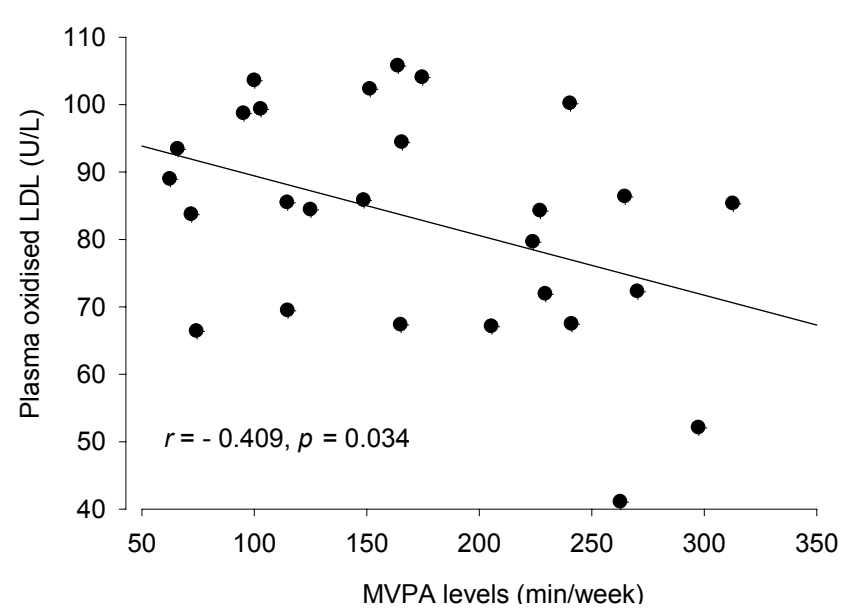

Fig. 1. The relationship between moderate to vigorous physical activity (MVPA) levels and fasting plasma oxidised low-density lipoprotein (LDL) concentrations in older adults $(n=27)$.

\section{Discussion}

To the best of our knowledge, the present study is the first to examine the relationship between the amount of physical activity and oxidised LDL in older adults. The main finding of the present study is that fasting plasma oxidised LDL concentrations were negatively correlated with the amount of physical activity, indicating that regularly active older individuals, at least in our study population, exhibited lower oxidised LDL concentrations.

The present study advances previous observations of lower oxidised LDL in aerobically trained middle-aged individuals ${ }^{7)}$ and demonstrates that this finding is also observed in "older" adults. This is significant because young adults have lower oxidised LDL concentrations than older adults ${ }^{10)}$ and elevated oxidised LDL concentrations are predictive of coronary heart disease ${ }^{4,14)}$, acute coronary syndromes ${ }^{15)}$, and metabolic syndrome ${ }^{5)}$. Importantly, it is worth noting that a negative association was observed between the amount of physical activity and plasma oxidised LDL concentrations, even in "healthy older" adults. These findings may imply that being physically active is important to reduce the potential risk of atherosclerotic cardiovascular disease in older adults if they can maintain such a status throughout daily life.

As in the design of the present study, it is not possible to determine the mechanisms responsible for the oxidised LDL-lowering effects of regular physical activity - our study aim was not to explore the mechanisms responsible for the oxidised LDL-lowering effects of regular physical activity. Nonetheless, likely

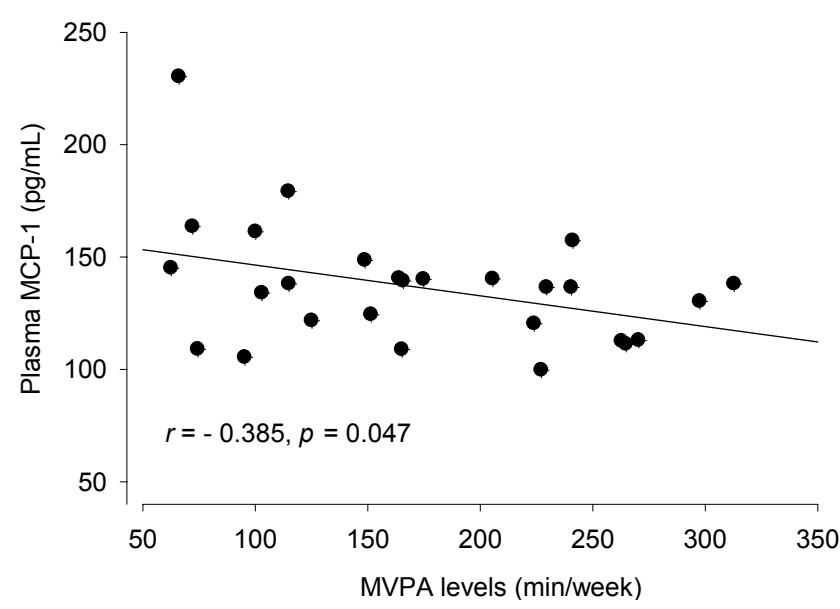

Fig. 2. The relationship between moderate to vigorous physical activity (MVPA) levels and fasting plasma monocyte chemoattractant protein-1 (MCP-1) concentrations in older adults $(n=27)$.

factors contributing to lowered oxidised LDL concentrations in physically active older individuals are better maintenance of the prooxidant/antioxidant balance by maintaining lower amounts of free radicals and thus reducing membrane lipid peroxidation through regular physical activity ${ }^{16}$. However, we have no direct data to support this. Thus, such speculation requires a further physical activity intervention study. Regarding another potential factor affecting oxidised LDL in physically active individuals, some researchers have suggested that HDL-C inhibits LDL oxidation ${ }^{17)}$. Our data demonstrated that although there was a positive relationship between the amount of physical activity and HDL-C, we failed to find a relationship between HDL-C and oxidised LDL (data not shown).

In the present study, we also observed that the amount of physical activity was negatively correlated with plasma MCP-1 concentrations. This is another interesting finding of our study since oxidatively modified LDL stimulates endothelial cells to produce MCP-1, which recruits circulating monocytes to vessel wall tissue ${ }^{18)}$. Our findings are consistent with data from a previous study examining the relationship between physical activity levels and plasma MCP-1 concentrations in middle-aged type 2 diabetic and coronary artery disease patients ${ }^{19)}$. However, our study is not consistent with a study of young trained adults $^{20)}$, which demonstrated that circulating concentrations of MCP-1 were similar between highly trained athletes $(65.0 \pm 26.2 \mathrm{pg} / \mathrm{mL})$ and their sedentary peers $(71.7 \pm 25.1 \mathrm{pg} / \mathrm{mL}$, means $\pm \mathrm{SD}$. $)$. This discrepancy may be due to differences in age between the studies since circulating concentrations of MCP-1 
Table 2. Correlations between the amount of moderate to vigorous physical activity and other blood parameters

\begin{tabular}{lrc}
\hline \multicolumn{1}{c}{ Variable } & $r^{\S}$ & $p$ \\
\hline TC & -0.001 & 0.995 \\
Triacylglycerol & -0.415 & 0.031 \\
HDL-C & 0.405 & 0.036 \\
LDL-C & -0.170 & 0.397 \\
Glucose & -0.160 & 0.425 \\
Insulin & -0.482 & 0.011 \\
Leptin & 0.115 & 0.567 \\
CRP & 0.198 & 0.323 \\
sVCAM-1 & -0.182 & 0.363 \\
Apolipoprotein(a) & 0.061 & 0.761 \\
Adiponectin & 0.336 & 0.087 \\
\hline
\end{tabular}

§; Pearson's correlation coefficient; TC, total cholesterol; HDL-C, high-density lipoprotein cholesterol; LDL-C, low-density lipoprotein cholesterol; CRP, C-reactive protein; sVCAM-1, soluble vascular cell adhesion molecule- 1 .

were higher in the present study $(136.4 \pm 27.0$, mean \pm SD., range $99.7-230.3 \mathrm{pg} / \mathrm{mL}$ ) compared with a previous study of both highly trained athletes and sedentary individuals, suggesting that the previous study may not have been sensitive enough to detect differences in MCP-1 concentrations between groups ${ }^{20)}$. Nonetheless, taken together with the results from the present study, a sedentary lifestyle may lead to accelerate atherosclerotic lesion formation and thus the development of cardiovascular disease.

We have also shown that the amount of physical activity was negatively correlated with serum triacylglycerol concentrations or plasma insulin concentrations. Indeed, insulin/triacylglycerol itself is a strong independent risk factor for cardiovascular disease ${ }^{21,22)}$, suggesting that regular physical activity might reduce the risk of cardiovascular disease by keeping insulin/ triacylglycerol concentrations low. The present study also found that the amount of physical activity was positively correlated with serum HDL-C concentrations. These observations are consistent with data from the meta-analyses ${ }^{23)}$, indicating that physically active individuals have significantly higher concentrations of HDL-C than their sedentary counterparts. Also, meta-analysis of previous studies suggested that a higher training volume (i.e. duration $\times$ intensity) was necessary to increase HDL-C ${ }^{24)}$.

The present study had limitations. First, based on its cross-sectional design, the present findings are inherently limited in their ability to eliminate causal relationships between plasma oxidised LDL concentrations and the amount of physical activity. Second, the sample size is small, which limited our ability to determine the significance. Data from follow-up studies with a large sample size are needed to determine the effectiveness of regular physical activity, if any, on lowering circulating concentrations of oxidised LDL in older adults.

\section{Conclusion}

The present study is the first to demonstrate, in a cross-sectional study, that lower concentrations of oxidised LDL were associated with a higher amount of physical activity in older adults. Although multiple regression analysis showed that the amount of MVPA is not an independent predictive factor of fasting plasma oxidised LDL concentrations, our data suggest that the oxidative modification of LDL may be prevented through regular physical activity. It would be interesting to investigate whether physical activity interventions can be used to slow the progression of atherosclerosis in older adults.

\section{Acknowledgements}

JHP supervised data collection, performed blood and data analysis, and wrote the first draft of the manuscript. MM conceived the study, obtained funding, recruited participants, assisted JHP with data collection and blood analysis, and edited the manuscript. MT was involved in the recruitment of participants and assisted JHP with data collection and blood analysis. $\mathrm{KH}, \mathrm{KT}$ and HSK were involved in the recruitment of participants and assisted JHP with data collection. KS performed venous blood collection and provided guidance and assistance to JHP during the study. YN obtained funding and provided guidance and assistance to JHP during the study. All authors contributed to the writing of the manuscript.

\section{Sources of Funding}

This study was supported by grants from the Ministry of Education, Culture, Sports Science and Technology of Japan, a Grant-in-Aid from the Global COE program "Sport Science for the Promotion of Active Life" (2010-2011) (to YN), the Health Promotion Foundation (to MM) and the Japan Health Foundation for the Prevention of Chronic Diseases and the Improvement of Quality of Life of Patients (to MM).

\section{Conflict of Interest}

None of the authors have any conflicts of interest regarding any aspect of this research. 


\section{References}

1) Itabe $H$, Ueda M: Measurement of plasma oxidized lowdensity lipoprotein and its clinical implications. J Atheroscler Thromb, 2007; 14: 1-11

2) Suzuki T, Kohno H, Hasegawa A, Toshima $S$, Amaki T, Kurabayashi M, Nagai R, Suzuki T, Amaki T, Nagai R, Hasegawa A, Toshima S, Kurabayashi MH, Shimada K, Nakamura $\mathrm{H}$, Teramoto T, Yamaguchi H, Nishiyama S, Takahashi H, Michishita I, Sugano Z, Konoshi K: Clinical Trial of Oxidized LDL (CTOL) investigators: Diagnostic implications of circulating oxidized low density lipoprotein levels as a biochemical risk marker of coronary artery disease. Clin Biochem, 2002; 35: 347-353

3) Ehara S, Ueda M, Naruko T, Haze K, Itoh A, Otsuka M, Komatsu R, Matsuo T, Itabe H, Takano T, Tsukamoto Y, Yoshiyama M, Takeuchi K, Yoshikawa J, Becker AE: Elevated levels of oxidized low density lipoprotein show a positive relationship with the severity of acute coronary syndromes. Circulation, 2001; 103: 1955-1960

4) Holvoet P, Mertens A, Verhamme P, Bogaerts K, Beyens G, Verhaeghe R, Collen D, Muls E, Van de Werf F: Circulating oxidized LDL is a useful marker for identifying patients with coronary artery disease. Arterioscler Thromb Vasc Biol, 2001; 21: 844-848

5) Holvoet P, Lee DH, Steffes M, Gross M, Jacobs DR: Association between circulating oxidized low-density lipoprotein and incidence of the metabolic syndrome. JAMA, 2008; 299: 2287-2293

6) Yamagishi S, Matsuoka H, Kitano S, Hibi N, Jinnouchi Y, Umei H, Iida S, Takenaka K, Matsui T, Nakamura K, Imaizumi T: Elevated circulating oxidized LDL levels in Japanese subjects with the metabolic syndrome. Int J Cardiol, 2007; 118: 270-272

7) Vinagre CG, Ficker ES, Finazzo C, Alves MJ, Angelis K, Irigoyen MC, Negrão CE, Maranhão RC: Enhanced removal from the plasma of LDL-like nanoemulsion cholesteryl ester in trained men compared with sedentary healthy men. J Appl Physiol, 2007; 103: 1166-1171

8) Ziegler S, Schaller G, Mittermayer F, Pleiner J, Mihaly J, Niessner A, Richter B, Steiner-Boeker S, Penak M, Strasser B, Wolzt M: Exercise training improves low-density lipoprotein oxidability in untraned subjects with coronary artery disease. Arch Phys Med Rehabil, 2006; 87: 265269

9) Vuorimaa $T$, Ahotupa M, Irjala K, Vasankari $T$ : Acute prolonged exercise reduces moderately oxidized LDL in healthy men. Int J Sports Med, 2005; 26: 420-425

10) Cakatay U, Kayali R, Uzun H: Relation of plasma protein oxidation parameters and paraoxonase activity in the ageing population. Clin Exp Med, 2008; 8: 51-57

11) Chen C, Jerome GJ, Laferriere D, Young DR, Vollmer WM: Procedures used to standardize data collected by
RT3 triaxial accelerometers in a large-scale weight-loss trial. J Phys Act Health, 2009; 6: 354-359

12) Matthews CE, Chen KY, Freedson PS, Buchowski MS, Beech BM, Pate RR, Troiano RP: Amount of time spent in sedentary behaviors in the United States, 2003-2004. Am J Epidemiol, 2008; 167: 875-881

13) Kumahara H, Schutz Y, Ayabe M, Yoshioka M, Yoshitake Y, Shindo M, Ishii K, Tanaka H: The use of uniaxial accelerometry for the assessment of physical-activityrelated energy expenditure: a validation study against whole-body indirect calorimetry. Br J Nutr, 2004; 91: 235-243

14) Meisinger C, Baumert J, Khuseyinova N, Loewel H, Koenig W: Plasma oxidized low-density lipoprotein, a strong predictor for acute coronary heart disease events in apparently healthy, middle-aged men from the general population. Circulation, 2005; 112: 651-657

15) Holvoet P, Vanhaecke J, Janssens S, Van de Werf F, Collen D: Oxidized LDL and malondialdehyde-modified LDL in patients with acute coronary syndromes and stable coronary artery disease. Circulation, 1998; 98: 1487-1494

16) Li LJ: Antioxidant enzyme response to exercise and aging. Med Sci Sports Exerc, 1993; 25: 225-231

17) Mackness MI, Durrington PN: HDL, its enzymes and its potential to influence lipid peroxidation. Atherosclerosis, 1995; 115: 243-253

18) Shin WS, Szuba A, Rockson SG: The role of chemokines in human cardiovascular pathology: enhanced biological insights. Atherosclerosis, 2002; 160: 91-102

19) Harsimran K, Singh AA, Guruvinder $S$, Sharda $S$, Vasudha S: Plasma monocyte chemoattractant protein-1 as risk marker in type 2 diabetes mellitus and coronary artery disease in North Indians. Diab Vasc Dis Res, 2009; 6: 288-290

20) Lira FS, Rosa JC, Pimentel GD, Souza HA, Caperuto EC, Carnevali LC, Seelaender M, Damasa AR, Oyama LM, Mello MT, Santos RV: Endotoxin levels correlate positively with a sedentary lifestyle and negatively with highly trained subjects. Lipids Health Dis, 2010; 9: 82

21) Després JP, Lamarche B, Mauriège P, Cantin B, Dagenais GR, Moorjani S, Lupien PJ: Hyperinsulinemia as an independent risk factor for ischemic heart disease. $\mathrm{N}$ Engl J Med, 1996; 334: 952-957

22) Hardman AE, Stensel DJ: Physical activity and Health: The evidence explained. 2nd Edition, Routledge, Abingdon, Oxon, 2009

23) Hata Y, Nakajima K: Life-style and serum lipids and lipoproteins. J Atheroscler Thromb, 2007; 7: 177-197

24) Kodama S, Tanaka S, Saito K, Shu M, Sone Y, Onitake F, Suzuki E, Shimano H, Yamamoto S, Kondo K, Ohashi Y, Yamada N, Sone H: Effect of aerobic exercise training on serum levels of high-density lipoprotein cholesterol: a meta-analysis. Arch Inern Med, 2007; 167: 999-1008 